\title{
Composition and organization of the sagittal stratum in the human brain: a fiber dissection study
}

\author{
Igor Lima Maldonado, MD, PhD, ${ }^{1-3}$ Christophe Destrieux, MD, PhD, 1,2 \\ Eduardo Carvalhal Ribas, MD, PhD, ${ }^{4}$ Bruna Siqueira de Abreu Brito Guimarães, MD, ${ }^{5}$ \\ Patrícia Pontes Cruz, MD, ${ }^{6}$ and Hugues Duffau, MD, PhD 7,8
}

\begin{abstract}
1 UMR 1253, iBrain, Université de Tours, Inserm, Tours, France; ${ }^{2} \mathrm{CHRU}$ de Tours, France; ${ }^{3}$ Le Studium Loire Valley Institute for Advanced Studies, Orléans, France; ${ }^{4}$ Department of Neurology, Discipline of Neurosurgery, University of São Paulo Medical School (FMUSP), São Paulo, Brazil; ${ }^{5}$ Faculdade de Medicina da Bahia, Universidade Federal da Bahia, Salvador, Brazil; ${ }^{6}$ Departamento de Ciências da Vida, Universidade do Estado da Bahia, Salvador, Brazil; ${ }^{2}$ Department of Neurosurgery, Gui de Chauliac Hospital, Montpellier University Medical Center, Montpellier, France; and ${ }^{8}$ INSERM-1051, Team 4, Saint-Eloi Hospital, Institute for Neurosciences of Montpellier, France
\end{abstract}

\begin{abstract}
OBJECTIVE The sagittal stratum is divided into two layers. In classic descriptions, the stratum sagittale internum corresponds to optic radiations (RADs), whereas the stratum sagittale externum corresponds to fibers of the inferior longitudinal fasciculus. Although advanced for the time it was proposed, this schematic organization seems simplistic considering the recent progress on the understanding of cerebral connectivity and needs to be updated. Therefore, the authors sought to investigate the composition of the sagittal stratum and to detail the anatomical relationships among the macroscopic fasciculi.
\end{abstract}

METHODS The authors performed a layer-by-layer fiber dissection from the superolateral aspect to the ventricular cavity in 20 cadaveric human hemispheres.

RESULTS Diverse bundles of white matter were observed to contribute to the sagittal stratum and their spatial arrangement was highly consistent from one individual to another. This was the case of the middle longitudinal fasciculus, the inferior fronto-occipital fasciculus, the RADs, and other posterior thalamic radiations directed to nonvisual areas of the cerebral cortex. In addition, small contributions to the sagittal stratum came from the anterior commissure anteriorly and the inferior longitudinal fasciculus inferiorly.

CONCLUSIONS A general model of sagittal stratum organization in layers is possible, but the composition of the external layer is much more complex than is mentioned in classic descriptions. A small contribution of the inferior longitudinal fasciculus is the main difference between the present results and the classic descriptions in which this bundle was considered to entirely correspond to the stratum sagittale externum. This subject has important implications both for fundamental research and neurosurgery, as well as for the development of surgical approaches for the cerebral parenchyma and ventricular system.

https://thejns.org/doi/abs/10.3171/2020.7.JNS192846

KEYWORDS neuroanatomy; temporal lobe; white matter; visual pathways; lateral ventricles; anatomy

$\mathrm{T}$ HE first descriptions of the sagittal stratum (SS) by Heinrich Sachs ${ }^{1,2}$ were briefly mentioned by Déjerine in his classic book, Anatomie des Centres Nerveux. ${ }^{3,4}$ Sachs used several histological techniques and studied fiber degeneration to describe this structure. The term "sagittal strata" was employed to name the white matter layers located around the atrium, occipital horn, and callosal fibers. Sachs described two layers: the stratum sagittale internum and the stratum sagittale externum. The stratum sagittale internum essentially corresponded to the optic radiations (RADs), whereas the stratum sagittale externum only included fibers of the inferior longitudinal

ABBREVIATIONS AF = arcuate fasciculus; iFOF = inferior fronto-occipital fasciculus; ILF = inferior longitudinal fasciculus; MdLF = middle longitudinal fasciculus; RAD = optic radiation; SLF = superior longitudinal fasciculus; $S S$ = sagittal stratum; UF = uncinate fasciculus.

SUBMITTED October 18, 2019. ACCEPTED July 17, 2020.

INCLUDE WHEN CITING Published online January 8, 2021; DOI: 10.3171/2020.7.JNS192846. 
fasciculus (ILF). The tapetum, made up of callosal fibers, was not included in the original description. Although advanced for that time, this anatomy needs to be revisited and updated as it was described prior to the development of Klingler's technique, and far before the description of the inferior fronto-occipital fasciculus (iFOF) and middle longitudinal fasciculus (MdLF). ${ }^{5,6}$

The term "sagittal stratum" was adopted by Ludwig and Klingler in their Atlas Cerebri Humani published in 1956 . This term is still used even if the division originally proposed by Sachs ${ }^{1}$ is not. Although the term became widespread, its exact meaning remains imprecise and varies among authors. ${ }^{1,7-11}$ For instance, similarly to Sachs, Fernández-Miranda et al. also consider the SS to contain an internal and an external layer. ${ }^{12}$ Nevertheless, the reported contents of each layer are very different for these two authors: the stratum sagittale internum as described by Sachs in 1892 includes the RADs, whereas the internal layer of the SS was reported by Fernández-Miranda et al. in 2008 to be composed of the parietopontine and occipitopontine fibers. Similarly, the stratum sagittale externum (Sachs ${ }^{1}$ ) only contains ILF fibers, whereas according to Fernández-Miranda et al., the external layer of the SS only includes the RADs. According to their definition, the ILF, $\mathrm{iFOF}$, and posterior limb of the anterior commissure are located lateral to the SS.,2,12

In the present study, we used fiber dissection to investigate the composition of the SS and precisely describe the anatomical relationships among its macroscopic fasciculi in cadaveric human brains. This topic has important implications both for fundamental research and cognitive neurosciences, as well as for the development of surgical approaches for the cerebral parenchyma and ventricular system.

\section{Methods}

Twenty cerebral hemispheres obtained from the body donation programs of our institutions were employed to study SS fibers and their anatomical relationships. The bodies were prepared during the 24 hours following death.

We used a variant of the method described by Ludwig and Klingler, in which we fixed the specimen through bilateral carotid injections of formalin before its removal from the cranial cavity, a procedure that was demonstrated to be effective in other studies performed by our team..$^{13-15}$ In this procedure, $1.5 \mathrm{~L}$ of a $4 \%$ buffered formaldehyde solution was slowly injected over 60 minutes. Intracranial fixation preserves the original shape of the brain and reduces the risk of parenchymal damage during its extraction, given the greater firmness of the tissues. The brains were then fixed for at least 2 months in formalin solution and suspended by the basilar artery to avoid contact with the walls of the container. The bath was changed 24 hours later to remove excess blood in the solution. Each specimen was then frozen at $-20^{\circ} \mathrm{C}$ for at least 14 days and then slowly defrosted. To show the anatomical relationships of white matter tracts in the neighborhood of the lenticular nucleus with arteries from the anterior perforating space, the middle cerebral artery in each of four hemispheres was manually injected with red-colored, room tempera- ture vulcanizing silicone with a $20-\mathrm{mL}$ plastic syringe after selective catheterization and exhaustive lavage with physiological solution $(\mathrm{NaCl} 0.9 \%)$.

For exploration of the white matter and presentation of results, the 3D disposition of the main elements of the SS was exposed through progressive fiber dissection from the cortex of the lateral convexity to the ventricular system. After the gyri and sulci were studied, the gray matterrendered fragile and friable by congelation-was delicately separated from the white matter that remained firmer. The main instruments used were handmade wooden spatulas, used to lift and progressively remove the white matter bundles. An M655 surgical microscope (Wild Heerbrugg Co.) was used as well as microsurgical instruments when the naked eye and the single or binocular loupes were no longer sufficient.

\section{Results}

Since the precise definition of SS is unclear in the literature, we decided first to describe the various layers of white matter encountered during the dissection of the temporo-parieto-occipital junction from lateral to medial, before proposing a definition for the SS.

The gray matter, darkened and made friable by the preparation, was removed to expose the subcortical short association U-fibers. The latter were removed and the posterior part of the superior longitudinal fasciculus (SLF)/ arcuate fasciculus (AF) complex was exposed in the postero-superior portion of the temporal lobe and in the depth of the supramarginal gyrus, this area being the entry point for deeper dissection (Fig. 1). Then, the vertical (posterior) and arcuate components of the SLF/AF complex were progressively removed. The SLF/AF was clearly distinguishable from the deeper layers as their fibers were almost perpendicular: roughly vertical for the SLF/AF in this region and horizontal for deeper fibers.

Fibers of the ILF were only observed in the inferior margin of the preparation, intermingled with other elements of the region. Although most of the ILF fibers ran at the ventral aspect of the temporal and occipital lobe, some of them could indeed be dissected from the lateral aspect. At this stage, the temporal operculum was preserved so as not to eliminate the MdLF located superficially. The MdLF penetrated the superior temporal gyrus and planum temporale (Fig. 2A) as an organized fiber contingent progressively more compact from posterior to anterior. Posteriorly, albeit superficial, the MdLF was clearly intermingled with the surrounding fibers.

Running at a deeper level than the MdLF, the iFOF reached the temporal stem and the insula just posterior and dorsal to the limen insulae. Consequently, the superior temporal gyrus including the planum temporale, and the middle temporal gyrus had to be removed prior to its dissection (Fig. 2B). The posterior segment of the iFOF was partially covered laterally by the dorsal part of the MdLF. As the dissection progressed from the posterior to the middle segment (trunk) of the iFOF, the latter ran deeper to the MdLF, reaching the antero-inferior portion of the external and extreme capsules. The ventral part of the external and extreme capsules also contained the un- 
cinate fasciculus (UF), which was ventral to the anterior segment of the iFOF.

The optic radiations (RADs) are the next fiber contingent crossed by the dissection that originates from the lateral geniculate body and runs into the lateral wall of the atrium and occipital horn before reaching the lips of the calcarine fissure (Fig. 2C and D). As proposed by Peltier et al., the RAD can be subdivided into three segments from anterior to posterior. ${ }^{16}$ The first segment, or Meyer's loop, is the most anterior and forms a posteriorly concave curve in a lateral oblique plane in close relationships with the anterior commissure and, more anteriorly, the UF. The second segment, or body of the RAD, runs along the lateral aspect of the atrium and occipital horn. Finally, the third segment joins the lips of the calcarine fissure. As a whole, the RADs-from the thalamus to the cortex - can be considered as specific posterior thalamic radiations that share the same general organization as other adjacent thalamic radiations to the occipital and parietal cortices.

Finally, the tapetum was located medial to the RADs, containing fibers in a vertical organization (Figs. $2 \mathrm{~F}$ and $4 \mathrm{~F})$. This layer, which is derived from the corpus callosum, covers the lateral aspect of the ventricular atrium and occipital horn. This was the deepest white matter layer of the lateral wall of the atrium, only limited from the CSF by the ependyma (Fig. 2F).

In summary (Figs. 3 and 4), the lateral wall of the ventricular atrium comprised the following elements, from medial to lateral: the ventricular ependyma, the tapetum, the RADs, the iFOF, the MdLF, the posterior portion of the SLF/AF complex, the short association U-fibers, and the cerebral cortex. Some of these elements were also present in the lateral wall of the occipital ventricular horn. The ILF only occupied the ventrolateral aspect of the dissection, its fibers contributing little to the ventral aspect of the superficial layer of the SS. Most of these structures were only partially superimposed on a sagittal plane. The MdLF, narrower than the iFOF, covered only its upper portion. The iFOF, in turn, partially covered the RADs, which overflows ventrally at the level of Meyer's loop. The more ventrally located RADs thus exceed the area covered by the iFOF in its anterior-inferior portion and extended around the temporal horn. As it progressed forward, the iFOF moved away from it to reach the temporal stem and the insula. Therefore, a space was created behind the directional change of the UF fibers, ahead of the Meyer's loop and deep into the iFOF. This space was occupied by fibers from the anterior commissure, and opened in a fan of which the more caudal ones joined the SS. Although a large number of fibers were intermingled, this phenomenon varied according to the location considered. As a result, the layers could be separated at certain sites, whereas in other sites, the delamination procedure caused the fibers to rupture. For example, for the posterior segment of the iFOF, interspersed with the RADs, as both approach the occipital cortex; for the anterior commissure intermingled with those of the Meyer's loop; and for the fibers of the anterior commissure intermingled with those of the posterior portion of the UF (Fig. 3H and I). The major white matter tracts are highlighted with colored lines in Supplementary Figs. 1-3.
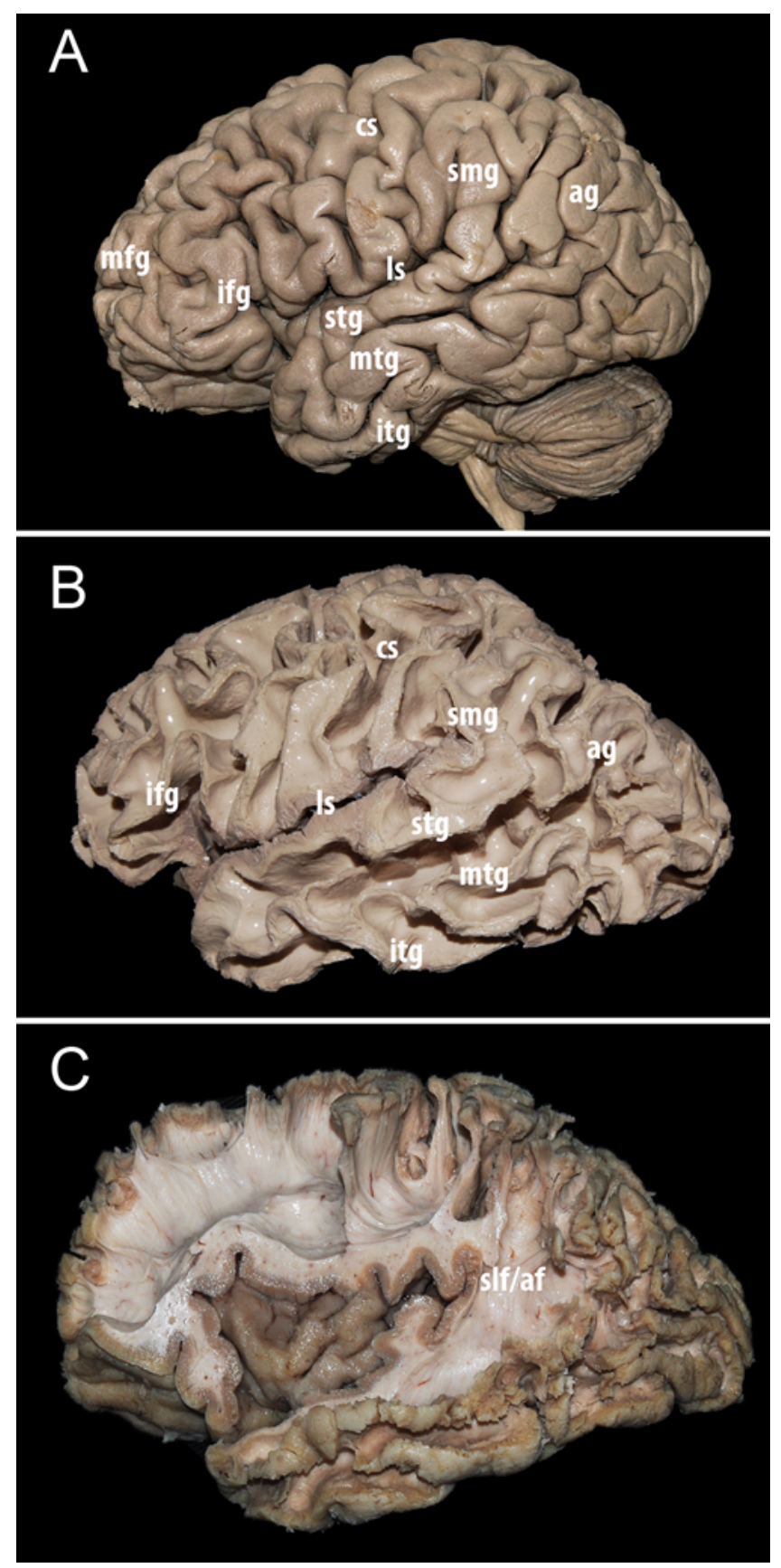

FIG. 1. Superficial dissection. A: A cadaveric human cerebral specimen prepared for fiber dissection using a variant of the Klingler method. The gray matter was dark and friable due to freezing. B: Subcortical white matter containing short association U-fibers. Delicate removal of the cerebral cortex exposes this layer of white matter at the depth of all sulci. It is smooth in the depth but rough at the top of each gyri, given the richness of the fiber terminations. C: Exposure of the posterior part of the superior longitudinal fasciculus/arcuate fasciculus (slf/af) complex. af $=$ arcuate fasciculus; ag = angular gyrus; cs = central sulcus; ifg = inferior frontal gyrus (triangular portion); itg = inferior temporal gyrus; ls = lateral sulcus; $\mathrm{mfg}=$ middle frontal gyrus; $\mathrm{mtg}$ = middle temporal gyrus; slf = superior longitudinal fasciculus; smg = supramarginal gyrus; stg = superior temporal gyrus. Figure is available in color online only. 

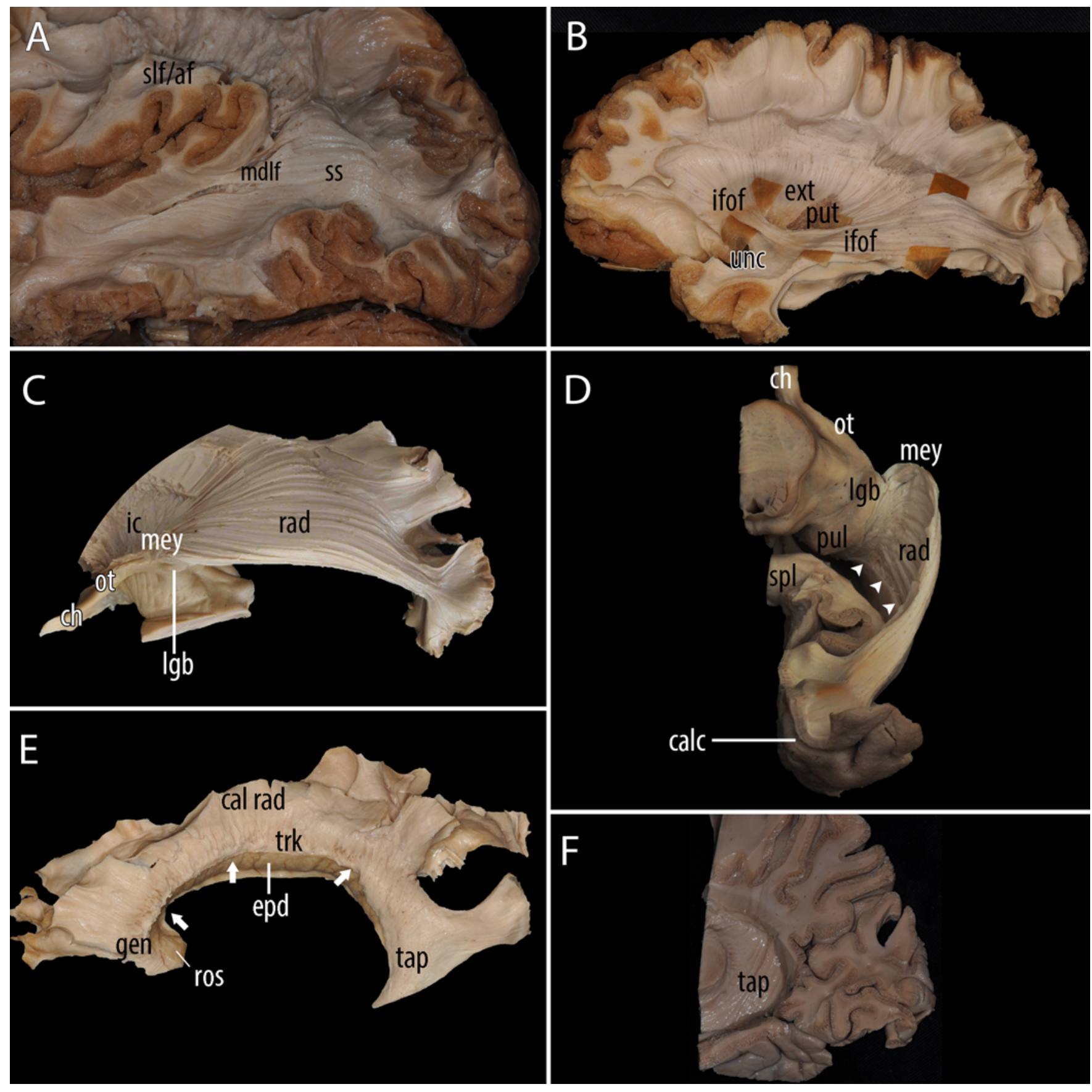

FIG. 2. Progressive exposure and dissection of the white matter fasciculi that run into the sagittal stratum (ss), medial to the superior longitudinal fasiculus (slf)/arcuate fasciculus (af). A: Middle longitudinal fasciculus (mdlf), lateral view. Exposure of the outermost layer of the ss after ablation of the elements of the sif/af complex, which is lateral to it, and before removal of the anterior temporal lobe. The mdlf fibers do not plunge into the deep parts of the temporal lobe as do those of the inferior fronto-occipital fasciculus (ifof) and optic radiations (rad). Instead, the mdlf fibers remain relatively superficial and penetrate the white matter of the temporal operculum. Reprinted with permission from Springer Nature: Maldonado IL, Zemmoura I, Destrieux C. Middle longitudinal fasciculus in the human brain from fiber dissection. In: Turgut M, Yurttaş C, Tubbs RS, eds. Island of Reil (Insula) in the Human Brain: Anatomical, Functional, Clinical and Surgical Aspects. Springer; 2018:71-75. B: Lateral view. The ifof is made of anterior and posterior segments linked by a narrower trunk. The uncinate (unc) fasciculus is ventral to the ifof trunk, and both constitute the ventral part of the external and extreme capsules. Dorsal to the ifof trunk, the dorsal part of the external capsule (ext) laterally limits the putamen (put). C: Lateral view, deeper dissection showing, from anterior to posterior: the optic chiasm (ch), the optic tract (ot) turning around the crus cerebri continuing to the internal capsule (ic), and the lateral geniculate body (lgb) and optic radiations (rad). The latter runs within the internal layer of the ss, deep to the mdlf and ifof before reaching the lips of the calcarine fissure in the occipital lobe. Meyer's loop (mey) is the anterior part of the rad. D: Inferior view on the same specimen as in panel C; the lateral ventricle was widely opened, and the dissection was performed from within its cavity; first, the ependyma was removed, followed by the tapetum, a thin layer of fibers roughly vertically oriented. The optic chiasm (ch), optic tract (ot), lateral geniculate body (lgb), and pulvinar (pul) are exposed. Further posteriorly and medially, the splenium of the corpus callosum (spl) was sectioned on the midline. Fibers of the optic radiations (rad), which are part of the posterior thalamic radiations, reached the calcarine fissure (calc). These fibers ran into the medial aspect of the occipital lobe, as well as along their recurrent path around the temporal horn of the lateral ventricle, forming the temporal (Meyer's) loop (mey). The arrowheads indicate the section line of the ependyma and tapetum fibers. FIG. 2. (continued) $\rightarrow$ 
FIG. 2. E: Dissection of the corpus callosum, lateral view (different specimen from that shown in panels $C$ and $D$ ). The corpus callosum was isolated from the projection fibers and thus from the lateral wall of the majority of the ventricular cavity in a specimen where the two hemispheres were not dissociated. The rostrum (ros), genu (gen), body (trk), and dorsal callosal radiations (cal rad) were exposed. The splenium is not visible in this view because it is hidden by the tapetum (tap), which covers the wall of the atrium and part of the occipital horn. Dissection of the corpus callosum from the projection fibers and from the lateral wall of the frontal horn and body of the lateral ventricle imposes the section of a great number of ventral and rostral cal rad, which were intermingled with the fibers of the corona radiata. They were removed together with the corona radiata during removal of the lateral wall of the frontal horn and the body of the ventricle. This is the reason for the dotted cut-off appearance of the callosal white matter all along the region that corresponds to the roof of the lateral ventricle (arrows). Finally, the ependyma (epd) is seen under the body of the corpus callosum at the level of the roof of the lateral ventricle. F: Medial view of the lateral wall of the ventricular atrium after a parasagittal section and removal of the ventricular ependyma. The tapetum (tap) fibers are vertically arranged between the ependyma and posterior thalamic radiations/optic radiations. Figure is available in color online only.

\section{Discussion}

There is to date no consensus on the white matter bundles forming the SS or on its anatomical boundaries, which explains discrepancies across publications. In the present study, we used a variant of the fiber dissection technique introduced by Ludwig and Klingler ${ }^{7}$ to describe the white matter layers bordering the atrium of the lateral ventricle including association (MdLF, iFOF, and, to a lesser degree, ILF), projection (RADs, posterior thalamic radiations), and commissural (anterior commissure) fasciculi.

We propose a definition of the SS that includes all of the white matter located between the SLF/AF complex laterally and the tapetum medially. We do not include the SLF/AF and the tapetum into the definition since their orientations (roughly vertical) clearly differ from those of the SS bundles located between (roughly horizontal). The SS was limited anteriorly by the UF and ventrally by ventral fibers of the ILF. It was not possible to establish clear dorsal and posterior anatomical boundaries, as the SS was continuous with the corona radiata up to the dorsal and posterior borders of the cerebral hemisphere.

The SS internal layer contains RADs. The RADs correspond to projection fibers of the metathalamus and thus occupy the same plane of dissection as the posterior thalamic radiations. This layer extends around the temporal ventricular horn from the lateral geniculate body, but also from the posterolateral part of the pulvinar. This was first reported in 1907 by Gustave Roussy, ${ }^{17}$ who stated that "they follow a transverse and ascending course, traverse the posterior segment of the corona radiata, passing through the retro-lenticular segment of the internal capsule, to approach, at the posterior part of the thalamus, the pulvinar and the external nucleus." This observation was confirmed by Türe et al. using Klingler's technique. ${ }^{18}$ Moreover, Roussy, and more recently Fernández-Miranda et al., ${ }^{12}$ also drew attention to the fact that posterior thalamic radiations did not only project to the calcarine fissure, i.e., did not only include the RADs, but also wider projection fibers to the parieto-occipital cortex. The fact that the internal layer is composed only of RADs is widely but not unanimously accepted. ${ }^{12}$

A major difference between our observations and those of Sachs regards the composition of the external layer. According to Sachs, the outer layer (stratum sagittale externum) entirely corresponds to the ILF., ${ }^{1,2}$ The present data clearly demonstrated that this observation overestimates the participation of this fasciculus and disregards the participation of others. Most of the ILF fibers, more exposable from the ventral aspect of the cerebral hemisphere, were situated inferiorly or relatively close to the floor of the ventricle. Only a small fiber contingent curved laterally to enter the lateral ventricular wall. As a consequence, the ILF mainly contributed to the lower part of the SS, lateral to the RADs. Conversely, the lateral layer of the SS also contained two additional fasciculi which were not originally included, the MdLF and the iFOF, which partially intermingled with the ILF.

The MdLF forms the dorsal part of the lateral layer of the SS. Its location, penetration into the temporal operculum, and relationship to the medial aspect of the SLF/ AF complex are concordant with diffusion imaging and original radioisotopic tracing studies in animals. ${ }^{19,20}$ The main discrepancy, however, concerns the MdLF posterior terminations: while some diffusion imaging studies show that the MdLF ends within the angular gyrus, fiber dissection does not reproduce these results, ${ }^{13,19,21-23}$ as dissected horizontal fibers in the external layer of the SS can be followed in their anteroposterior trajectory beyond that gyrus. It can be hypothesized that evolutionary changes can be the cause of these differences. In phylogeny, the thick and widely developed SLF/AF complex in humans may have moved caudally to the point where the more internal horizontal fibers curve laterally. The partial overlap of the MdLF and iFOF fascicles was also highlighted by Di Carlo et al., who introduced the term "middle layer" of the SS for the iFOF. ${ }^{24}$

A precise knowledge of the composition and spatial organization of the SS is a keystone for several surgical applications, especially temporal lobectomies and approaches to the ventricular atrium or its lateral wall. ${ }^{8}$ Historically, morphological limits have been described to guide temporal lobectomies, which are still used when functional mapping techniques are not available. The rationale behind classic limits is in essence functional and is driven by the iFOF, ILF, and RADs, which partially run through the SS. With an oblique trajectory above the ventricular horn, the iFOF runs anteriorly and becomes progressively deeper in the temporal lobe, reaching the temporal stem, the insula, and finally the frontal lobe. ${ }^{25}$ Direct electrical stimulation has pointed to the role of the iFOF in language, especially semantics, in the so-called "dominant" hemisphere, , $^{86-28}$ but also to the implication of its function in nonverbal semantic cognition in the "nondominant" hemisphere. ${ }^{29}$ Moreover, when identified by subcortical mapping, it is a consistent landmark of where the anterolateral central (lenticulostriate) perforators from the middle cerebral artery start to be frequently encountered (Fig. 4A, B, D, and $\mathrm{H})$. The ILF is suggested to be an important component for interpretation and modulation of visual input. ${ }^{30}$ In the 


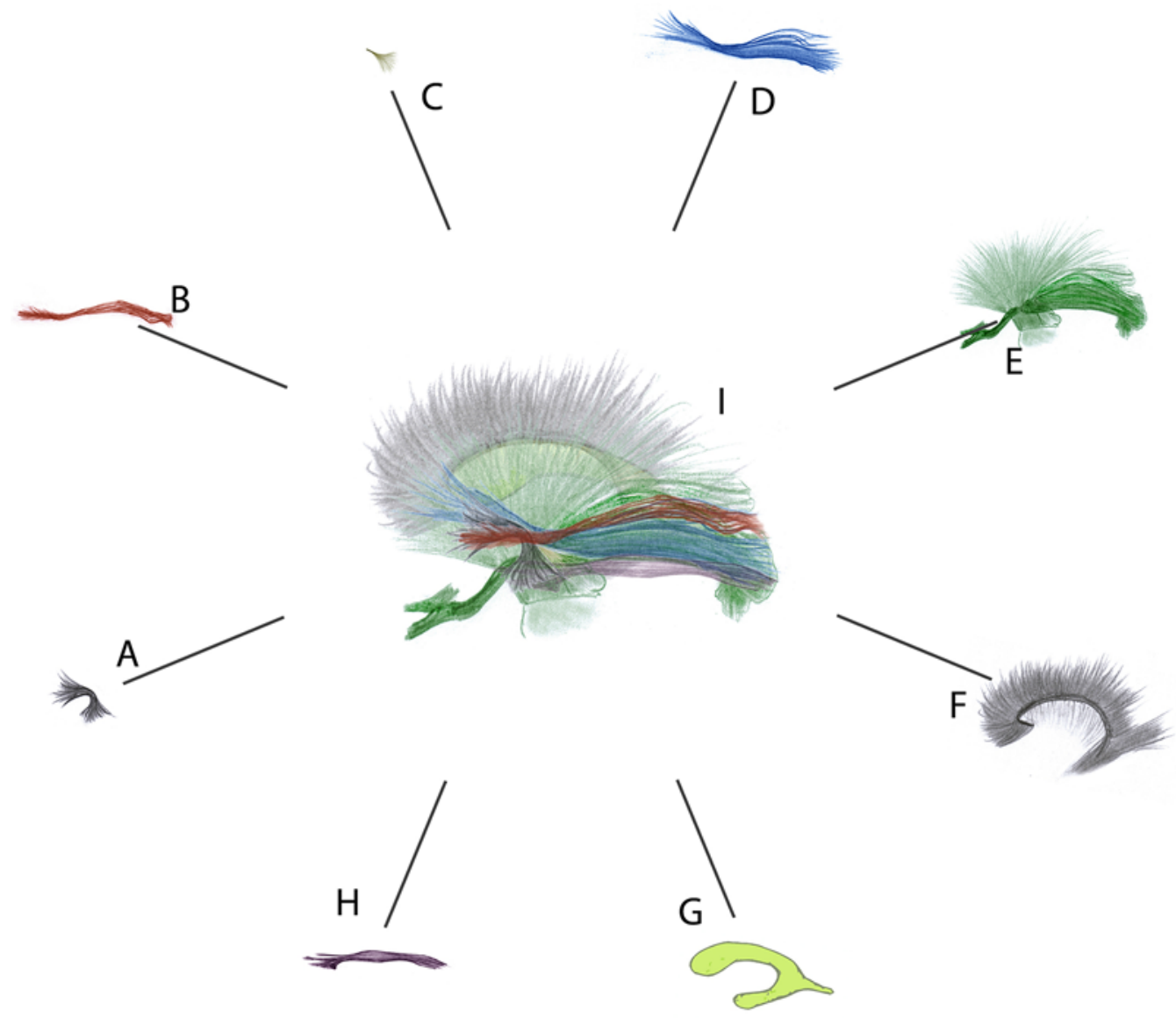

FIG. 3. Schematic representation of the general organization of the sagittal stratum. Except for the uncinate fasciculus, all elements are systematically located at least partially between the lateral ventricle and the superior longitudinal fasciculus/arcuate fasciculus complex. A: Uncinate fasciculus, the most anterior long association bundle in the temporal lobe. It limits the sagittal stratum anteriorly, and its posterior-most fibers partially cover it, intermingling with the anterior-most fibers of the anterior commissure. B: Middle longitudinal fasciculus. The posterior segment of this bundle is incorporated in the most superficial layer of the sagittal stratum, medial to the fibers of the superior longitudinal fasciculus/arcuate fasciculus complex. The middle longitudinal fasciculus covers the dorsal portion of the inferior fronto-occipital fasciculus. Its anterior segment, more superficial, penetrates the white matter of the superior temporal gyrus, deviating from the sagittal stratum without being covered anymore by the superior longitudinal fasciculus/arcuate fasciculus complex. C: Anterior commissure. The trunk of the anterior commissure crosses the antero-ventral portion of the lenticular nucleus through the so-called Gratiolet's canal, with a volume that is quite variable from one individual to another. The temporal fibers of this small bundle are oriented inferiorly and posteriorly. They are in great numbers covered by the inferior fronto-occipital fasciculus and uncinate fasciculus. Most often, they are not exposed until the inferior fronto-occipital tract has been removed. They partially cover Meyer's loop of the optic radiations, to which they are intimately intermingled, as they are to the most posterior fibers of the uncinate fasciculus. D: Inferior fronto-occipital fasciculus. The posterior segment of this bundle integrates the sagittal stratum and contributes greatly to the lateral wall of the ventricular atrium and occipital horn. As the inferior fronto-occipital fasciculus approaches the occipital cortex, its fibers intermingle with those of the optic radiations and the dorsal fibers of the inferior longitudinal fasciculus, which form the inferolateral edge of the dissection when the sagittal stratum is exposed. The middle segment of the inferior fronto-occipital fasciculus bends upward and medially to penetrate the temporal peduncle, covering most of the fibers of the anterior commissure, but not the most anterior portion of Meyer's loop. At this level, the bundle adopts a dorsal location in relation to the ventricular temporal horn, deviating from the inferior longitudinal fasciculus. E: Optic radiations (dark green) occupy the deep layer of the sagittal stratum. They are located within the same plane as other thalamic posterior radiations to extra calcarine destinations and blend into the group of projection fibers formed by the internal capsule and corona radiata (light green). The optic radiations cover the tapetum, a vertical layer of fibers from the corpus callosum. The optic radiations intermingle with the posterior fibers of the anterior commissure at the level of Meyer's loop, and with those of the inferior longitudinal and the fronto-occipital fasciculus in proximity to the occipital pole. F: The tapetum (from the corpus callosum). The corpus callosum contributes to the lateral ventricular wall with the tapetum, a layer of vertically oriented fibers insinuated between optic radiations and the ventricular ependyma. G: Lateral ventricle. The ventricular ependyma of the atrium and a large part of the temporal horn and occipital horn is directly related to the tapetum and is covered by the different layers of the sagittal stratum. $\mathrm{H}$ : Inferior longitudinal fasciculus. This fasciculus runs along the inferior aspect of the cerebral hemisphere, with most of its fibers being inferior to the ventricular cavity. When the sagittal stratum is exposed and the dissection plane is kept, it occupies the inferolateral angle of the dissection. I: Overview. The sagittal stratum gathers fibers from the structures shown in panels B-E and $\mathrm{H}$ (a synthetic overview is provided here: https://fibratlas.univ-tours.fr/?page_id=742). Figure is available in color online only. 

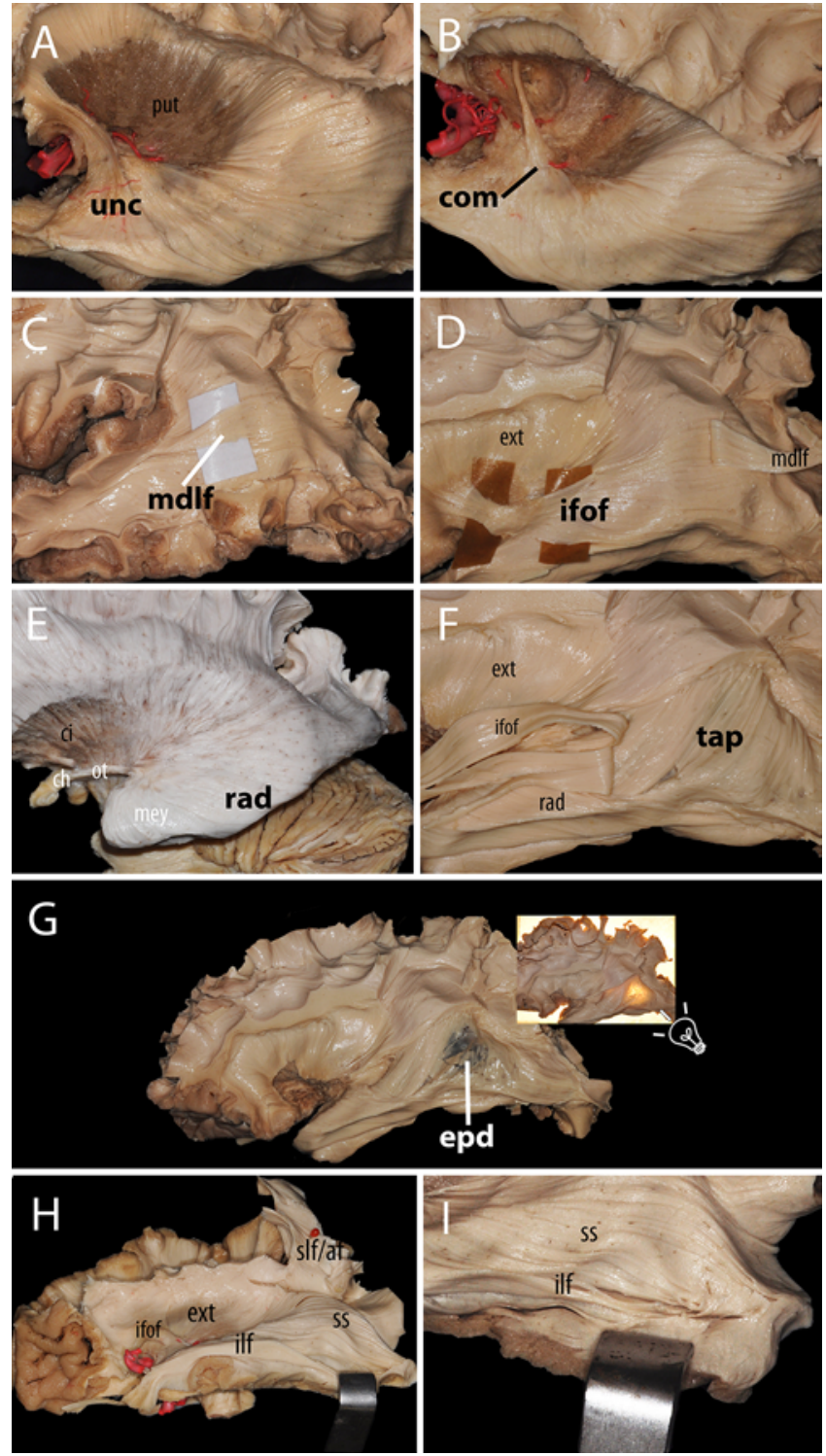

FIG. 4. Summary of white matter elements of the sagittal stratum (ss), neighboring structures, and anatomical relationships, such as those shown in Fig 3. A: Uncinate fasciculus (unc). Fibers of this bundle intermingle with those of the anterior commissure. No definitive limit is observed between the unc and inferior fronto-occipital fasciculus (ifof). The unc is part of the ventral external and extreme capsule, medial to the putamen (put). B: Anterior commissure (com). The most posterior fibers of the com are intermingled with the more anterior ones of Meyer's loop, are partially covered by the inferior fronto-occipital fasciculus, and occupy the space between the temporal portion of the uncinate fasciculus and temporal optic radiations. C: Middle longitudinal fasciculus (mdlf). D: Inferior fronto-occipital fasciculus (ifof). The trunks of the ifof and the uncinate fasciculus form the ventral part of the extreme and external (ext) capsules. The dorsal part of the ext has a radial pattern. The mdlf (retracted) is covering the posterior and dorsal part of the ifof. E: Optic radiations (rad). The optic chiasm (ch) is continued by the optic tract (ot), which turns around the crus cerebri, continuing ventrally to the internal capsule (ci). The ot is continued by the rad at the level of the lateral geniculate body (not visible). Meyer's loop (mey) contains the most anterior fibers of the optic radiations running into the temporal lobe. F: The tapetum (tap) is made of callosal fibers medially limiting the sagittal stratum. This dissection shows the internal (rad) and part of the external (ext) layer of the sagittal stratum (ifof, retracted mdlf, and inferior longitudinal fasciculus). FIG. 4. (continued) $\rightarrow$
FIG. 4. G: Ventricular ependyma (epd). H: Inferior longitudinal fasciculus (ilf), ventrolateral view. When the sagittal stratum (ss) is exposed, the ilf occupies the inferolateral border of the dissection. The superior longitudinal fasciculus/arcuate fasciculus (sIf/af) complex is dorsally retracted. I: Intermingling fibers between the ilf and posterior segment of the ifof and rad. Clear dissection of the ilf from surrounding tracts in the ss is impossible without causing these links to break. Figure is available in color online only.

dominant hemisphere, and in conjunction with the UF, the ILF forms an indirect ventral semantic stream. Resection of the anterior part of the ILF does nevertheless not induce semantic deficits, suggesting that it is compensated for by the iFOF. ${ }^{31}$ The same does not apply to posterior portions of the ILF, whose section can disconnect the visual word form area and induce severe and permanent reading disorders. ${ }^{32}$ This explains the classic posterior limit for temporal lobectomy aiming at preserving this area.

The RADs also have to be considered in planning a temporal lobectomy, since a superior quadrantanopsia can follow a lesion of Meyer's loop. They also have to be preserved in any approach to the atrium. The cortical projection of the visual field is well known: the superior lip of the calcarine fissure analyzes the lower visual fields of both eyes, whereas its inferior lip receives input from the upper visual fields. Cortical areas corresponding to the macula are located close to the occipital pole and have a bilateral representation, whereas the peripherical field of view is projected to the anterior calcarine. ${ }^{33}$

The visual field cortical areas get the following input from the geniculate body via the RADs: 1) fibers conveying inputs from the peripheral upper visual field are the most anterior ones within Meyer's temporal loop; they curve to form the ventral part of the SS internal layer and finally reach the anterior part of the ventral lip of the calcarine fissure; 2) fibers corresponding to the macular upper visual field run more posterior within Meyer's loop, then become dorsal to the previous fibers within the SS, and project more posteriorly (close to the occipital pole) at the ventral lip of the calcarine fissure; 3) fibers corresponding to the macular lower field run in the SS dorsal to fibers for the macular upper field and terminate in the upper calcarine lip also close to the occipital pole; and 4) fibers dedicated to the peripheral lower visual field are the most dorsal within the SS internal layer and progressively arch superiorly in the depth of the temporo-parietal junction and cover the atrium lateral to the tapetum, to finally end in the anterior part of the upper calcarine lip.

As a result, RADs in the SS correspond, from ventral to dorsal, to the peripheral upper, macular, and peripheral lower visual contralateral fields. This correspondence has to be taken into account during the planning of approaches to the temporo-parietal junction and ventricular cavity through its lateral wall. The orientation of fibers is also of interest, since incisions performed horizontally or with respect to the obliquity of fibers within the lateral ventricular wall may induce less disconnection.

The present results allowed systematization of the general organization of the SS, as well as important updates to data on the composition of the outer layer in relation to the original definitions by Sachs. ${ }^{1}$ Nevertheless, there are 
important areas of fiber crossing between the iFOF and RADs in the posterior portion of the ventricular atrium, between the anterior commissure and Meyer's loop, and along the ILF. As a consequence, our description is inevitably incomplete given the fact that groups of delicate and dispersed fibers may be difficult to dissect and partially destroyed during removal of the surrounding white matter. A more complete characterization of this region may emanate from the combination of complementary methods, such as ultra-high field MRI, optic coherence tomography, and polarized light imaging.

\section{Conclusions}

This study revisited the 3D organization of the white matter in the SS and shed light on certain unexplored points. Although a precise definition of its boundaries does not exist to date, we propose, based on the present results, that this region be delimited based on the surrounding white matter structures. The SS comprises the area between the SLF/AF laterally and the ventricular tapetum and ependyma medially. It is limited anteriorly by the uncinate fasciculus and inferiorly by the ILF, which is, in part, also a component of the SS. There is no precise delimitation of the superior and posterior limits as it is a continuous structure with the corona radiata and internal capsule.

This study also demonstrated the need for an update and redefinition of the classic SS composition. Diverse bundles of white matter contribute to the SS, and their spatial arrangement is highly consistent from one individual to another. That is the case for the MdLF, the iFOF, the RADs, and other posterior thalamic radiations directed to nonvisual areas of the cerebral cortex. The anterior commissure offers a small contribution to the SS anteriorly, as does the ILF inferiorly. Although a general model of SS organization in layers is possible, there are important sites of intermingling fibers that remain to be further explored.

\section{Acknowledgments}

This work was supported by the French National Agency of Research (The Fibratlas Project, ANR-14-CE17-0015) and the LE STUDIUM Loire Valley Institute for Research Studies, Orléans, France.

We would like to express our gratitude to the donors involved in the body donation program of the Association des dons du corps du Centre Ouest, Tours, who made this study possible by generously donating their bodies to science. We thank Daniel Bourry, photographer at the Université de Tours, for his technical assistance. We also thank Frank Meyer, Jean Marc Gory, Gerald Deluermoz, and Jean-Paul da Silva for their help in preparing the anatomic specimens.

\section{References}

1. Sachs H. Das Hemisphärenmark des Menschlichen Grosshirns. 1. Der Hinterhauptlappen. G Thieme; 1892.

2. Sachs H. Vorträge über Bau und Thätigkeit des Grosshirns und die Lehre von der Aphasie und Seelenblindheit für Aerzte und Studirende. Preuss \& Jünger; 1893.

3. Déjerine J. Anatomie des Centres Nerveux. Vol 1. Rueff et Cie; 1895.

4. Déjerine J. Anatomie des Centres Nerveux. Vol 2. Rueff et Cie; 1901.
5. Curran EJ. A new association fiber tract in the cerebrum. $J$ Comp Neurol. 1909;19(6):645-656.

6. Seltzer B, Pandya DN. Further observations on parietotemporal connections in the rhesus monkey. Exp Brain Res. 1984;55(2):301-312.

7. Ludwig E, Klingler J. Atlas Cerebri Humani. S Karger AG; 1956.

8. Chan-Seng E, Moritz-Gasser S, Duffau H. Awake mapping for low-grade gliomas involving the left sagittal stratum: anatomofunctional and surgical considerations. J Neurosurg. 2014;120(5):1069-1077.

9. Hosoya T, Adachi M, Yamaguchi K, Haku T. MRI anatomy of white matter layers around the trigone of the lateral ventricle. Neuroradiology. 1998;40(8):477-482.

10. Jellison BJ, Field AS, Medow J, et al. Diffusion tensor imaging of cerebral white matter: a pictorial review of physics, fiber tract anatomy, and tumor imaging patterns. AJNR Am J Neuroradiol. 2004;25(3):356-369.

11. Martino J, De Witt Hamer PC, Vergani F, et al. Cortexsparing fiber dissection: an improved method for the study of white matter anatomy in the human brain. J Anat. 2011; 219(4):531-541.

12. Fernández-Miranda JC, Rhoton AL Jr, Alvarez-Linera J, et al. Three-dimensional microsurgical and tractographic anatomy of the white matter of the human brain. Neurosurgery. 2008;62(6)(suppl 3):989-1028.

13. Maldonado IL, de Champfleur NM, Velut S, et al. Evidence of a middle longitudinal fasciculus in the human brain from fiber dissection. J Anat. 2013;223(1):38-45.

14. Martino J, Brogna C, Robles SG, et al. Anatomic dissection of the inferior fronto-occipital fasciculus revisited in the lights of brain stimulation data. Cortex. 2010;46(5):691-699.

15. Martino J, Vergani F, Gil Robles S, et al. New insights into the anatomic dissection of the temporal stem with special emphasis on the inferior fronto-occipital fasciculus: implications in surgical approach to left mesiotemporal and temporoinsular structures. Neurosurgery. 2010;66(3 Suppl Operative):4-12.

16. Peltier J, Travers N, Destrieux C, Velut S. Optic radiations: a microsurgical anatomical study. J Neurosurg. 2006;105(2): 294-300.

17. Roussy G. La Couche Optique (Etude Anatomique, Physiologique et Clinique). Le Syndrome Thalamique. G Steinheil; 1907.

18. Türe U, Yaşargil MG, Friedman AH, Al-Mefty O. Fiber dissection technique: lateral aspect of the brain. Neurosurgery. 2000;47(2):417-427.

19. Makris N, Papadimitriou GM, Kaiser JR, et al. Delineation of the middle longitudinal fascicle in humans: a quantitative, in vivo, DT-MRI study. Cereb Cortex. 2009;19(4):777-785.

20. Schmahmann JD, Pandya DN. Fiber Pathways of the Brain. Oxford University Press; 2006.

21. Makris N, Preti MG, Asami T, et al. Human middle longitudinal fascicle: variations in patterns of anatomical connections. Brain Struct Funct. 2013;218(4):951-968.

22. Maldonado IL, Zemmoura I, Destrieux C. Middle longitudinal fasciculus in the human brain from fiber dissection. In: Turgut M, Yurttaş C, Tubbs RS, eds. Island of Reil (Insula) in the Human Brain: Anatomical, Functional, Clinical and Surgical Aspects. Springer; 2018:71-75.

23. Menjot de Champfleur N, Maldonado IL, Moritz-Gasser S, et al. Middle longitudinal fasciculus delineation within language pathways: a diffusion tensor imaging study in human. Eur J Radiol. 2013;82(1):151-157.

24. Di Carlo DT, Benedetto N, Duffau H, et al. Microsurgical anatomy of the sagittal stratum. Acta Neurochir (Wien). 2019; 161(11):2319-2327.

25. Sarubbo S, De Benedictis A, Maldonado IL, et al. Frontal terminations for the inferior fronto-occipital fascicle: ana- 
tomical dissection, DTI study and functional considerations on a multi-component bundle. Brain Struct Funct. 2013; 218(1):21-37.

26. Duffau $\mathrm{H}$. The anatomo-functional connectivity of language revisited. New insights provided by electrostimulation and tractography. Neuropsychologia. 2008;46(4):927-934.

27. Duffau H, Capelle L, Sichez N, et al. Intraoperative mapping of the subcortical language pathways using direct stimulations. An anatomo-functional study. Brain. 2002;125(Pt 1): 199-214.

28. Duffau H, Gatignol P, Mandonnet E, et al. New insights into the anatomo-functional connectivity of the semantic system: a study using cortico-subcortical electrostimulations. Brain. 2005;128(Pt 4):797-810.

29. Herbet G, Moritz-Gasser S, Duffau H. Direct evidence for the contributive role of the right inferior fronto-occipital fasciculus in non-verbal semantic cognition. Brain Struct Funct. 2017;222(4):1597-1610.

30. Herbet G, Zemmoura I, Duffau H. Functional anatomy of the inferior longitudinal fasciculus: from historical reports to current hypotheses. Front Neuroanat. 2018;12:77.

31. Mandonnet E, Nouet A, Gatignol P, et al. Does the left inferior longitudinal fasciculus play a role in language? A brain stimulation study. Brain. 2007;130(Pt 3):623-629.

32. Zemmoura I, Herbet G, Moritz-Gasser S, Duffau H. New insights into the neural network mediating reading processes provided by cortico-subcortical electrical mapping. Hum Brain Mapp. 2015;36(6):2215-2230.

33. McFadzean R, Brosnahan D, Hadley D, Mutlukan E. Representation of the visual field in the occipital striate cortex. $\mathrm{Br} J$ Ophthalmol. 1994;78(3):185-190.

\section{Disclosures}

The authors report no conflict of interest concerning the materials or methods used in this study or the findings specified in this paper.

\section{Author Contributions}

Conception and design: Maldonado. Acquisition of data: Maldonado, Guimarães, Cruz. Analysis and interpretation of data: Maldonado, Destrieux, Ribas, Guimarães, Cruz. Drafting the article: Maldonado. Critically revising the article: all authors. Reviewed submitted version of manuscript: all authors. Administrative/technical/material support: Destrieux, Ribas, Duffau. Study supervision: Maldonado.

\section{Supplemental Information}

\section{Online-Only Content}

Supplemental material is available with the online version of the article.

Supplementary Figs. 1-3. https://thejns.org/doi/suppl/10.3171/ 2020.7.JNS192846.

\section{Correspondence}

Igor Lima Maldonado: iBrain, Université de Tours, Inserm, Tours, France.limamaldonado@univ-tours.fr. 